\title{
The connection between radio and Very High Energy in Active Galactic Nuclei: perspectives with CTA, the Cherenkov Telescope Array
}

\section{Hélène Sol ${ }^{1}$}

Observatoire de Paris, CNRS, LUTH

Place J. Janssen, 92195 Meudon, France

E-mail: helene.soleobspm. fr

\begin{abstract}
Current results on Active Galactic Nuclei (AGN) detected in the Very High Energy (VHE) range suggest that the elementary bricks of the VHE emission scenarios are now reasonably well identified. However, a global picture of the sources, describing the geometry and global dynamics of the VHE zone is not at all available at the moment. How VHE AGN fit into AGN unification schemes is also a critical open question. In the near future, a fruitful synergy between radio VLBI and VHE observations should help in understanding this puzzle, especially in the perspective of the Cherenkov Telescope Array (CTA), a major project of the next generation in ground-based gamma-ray astronomy. CTA will greatly improve the performances in sensitivity, effective energy coverage, field of view, angular and spectral resolution of present Imaging Atmospheric Cherenkov Telescopes and provide a wealth of detailed data on extreme non-thermal processes in AGN jets.
\end{abstract}

11th European VLBI Network Symposium \& Users Meeting

October 9-12, 2012

Bordeaux, France

${ }^{1}$ Speaker 


\section{Introduction}

Current ground-based Imaging Atmospheric Cherenkov Telescopes (Fig. 1) have now detected more than 50 Active Galactic Nuclei (AGN) at Very High Energy (VHE) in the TeV range. The fast variability observed in several sources implies from causality argument that the VHE emitting zones are often compact and likely located in the regions probed by VLBI in the radio range. Understanding the connection between VLBI and VHE properties of TeV AGN would be a significant step towards the development of a global scenario of non-thermal processes in AGN.
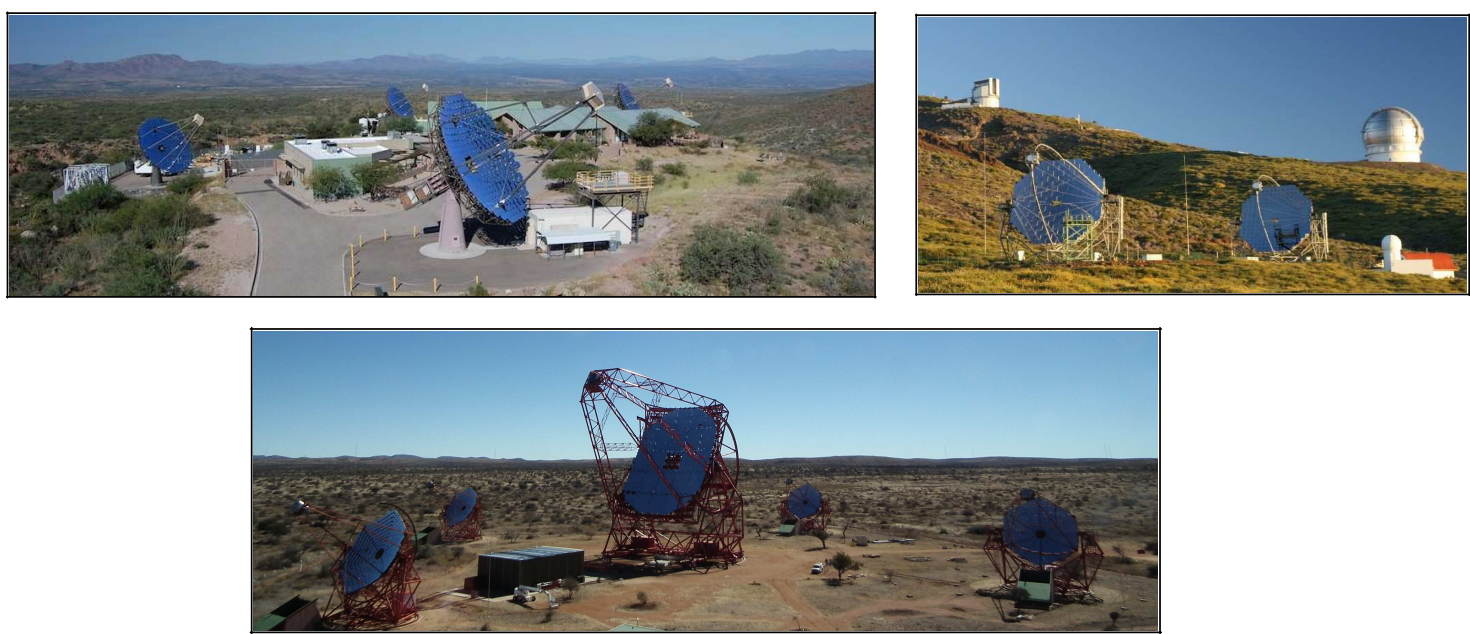

Figure 1. The three main current ground-based Imaging Atmospheric Cherenkov Telescopes, VERITAS (Very Energetic Radiation Imaging Telescope Array System, Arizona), MAGIC (Major Atmospheric Gamma Imaging Cherenkov Telescope, Canary island of La Palma) and HESS (High Energy Stereoscopic System, Namibia), with the new HESS II telescope (28 m).

\section{Active Galactic Nuclei seen at Very High Energy}

The current sample of AGN firmly detected in the range from $100 \mathrm{GeV}$ to $30 \mathrm{TeV}$ (Fig. 2) includes 47 blazars, 4 radiogalaxies (M87, Cen A, NGC 1275 and IC130), and one AGN of unknown type (VER J0521+211), with redshift from 0.00183 (Cen A) to 0.536 (3C 279). The detection at $5 \sigma$ of KUV 00311-1938 by HESS might provide a new record in redshift if its value of $\mathrm{z}=0.61$ is confirmed [2,3]. Only point-like sources have been detected up to now. Among the $47 \mathrm{TeV}$ blazars, there are $34 \mathrm{HBL}$ (High frequency peak BL Lacs), 4 IBL (Intermediate frequency peak BL Lacs), namely 3C66A, W Comae, PKS 1424+240 and 1ES1440+122, 4 LBL (Low frequency peak BL Lacs), namely BL Lac itself, AP Lib, 1ES1215+303 and S5 0716+714, 3 FSRQ (Flat Spectrum Radio Quasars), namely 3C 279, PKS 1510-089 and 4C +21.35 , and 2 still unclassified BL Lacs.

Typically $90 \%$ of the AGN discovered at VHE are presumably beamed sources with relativistic jets pointing to the observer. The TeV sample is highly biased towards sources with strong Doppler boosting where the apparent VHE flux is enhanced by a factor $\sim \delta^{4}$. High Doppler factors $\delta$ also help to accommodate fast variability and to avoid strong intrinsic absorption. 


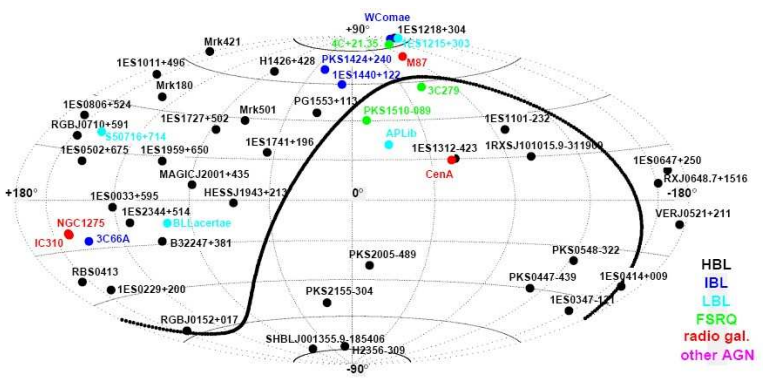

Figure 2. Distribution of the current sample of AGN detected at very high energies shown in galactic coordinates (from TeVCat catalog, October 2012, see [1]).

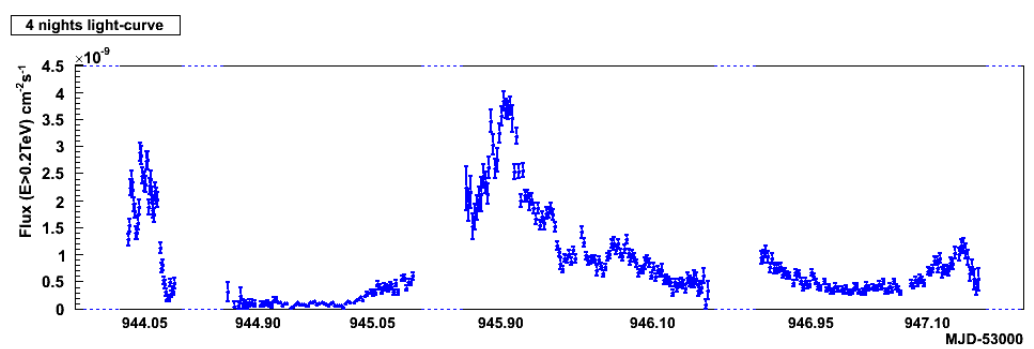

Figure 3. Integrated flux versus time during the very active state of PKS 2155-304 in July 2006, showing two big flares and variability on all time scales from a few minutes to several days (see [4] for more details).
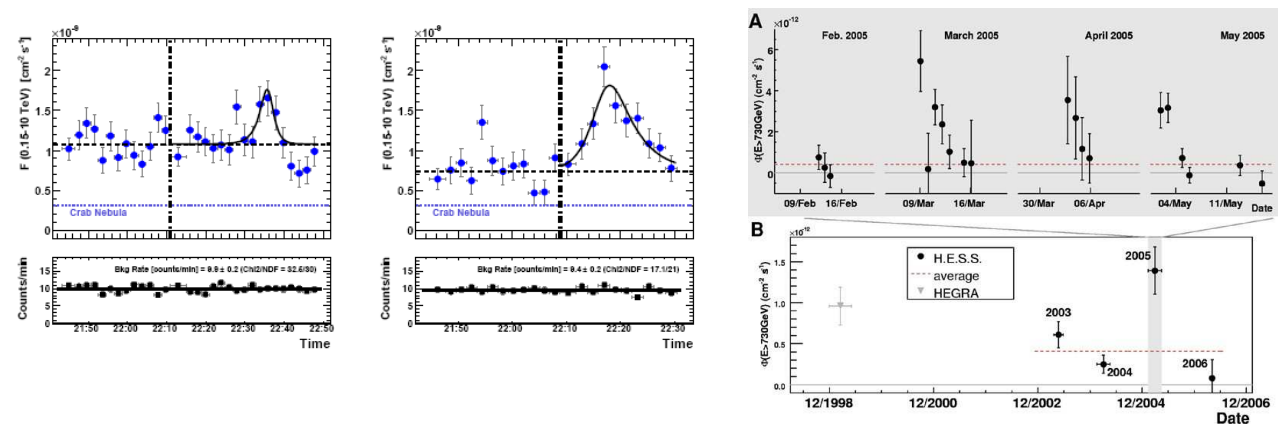

Figure 4. Examples of fast variability detected in Mrk 501 and M87 [5, 6].

VHE blazars are mostly highly variable sources, with variability detected on all timescales, from minutes to years, despite a usually rather poor time coverage (Figs. 3, 4 and 11). Fast variability on typical timescale $t_{v a r}$ implies in several cases a very small size $R$ for the VHE emitting zones. From causality argument: $\mathrm{R}<\mathrm{ct}_{\mathrm{var}} \delta /(1+\mathrm{z})$, which becomes critical compared to the Schwarzschild radius of central black holes, even for high $\delta$, in several sources such as PKS 2155-304, Mrk 501 and M87. The current VHE sample is likely biased towards AGN active states because of the limit in sensitivity of the instruments, and also because of a frequent strategy of observations of targets of opportunity after multi-wavelength (MWL) alerts for flares. However, stationary and quiescent states have been probably detected for some of the brightest sources as PKS 2155-304 (Fig. 5). AGN are large band emitters and MWL data are mandatory to constrain their spectral energy distribution (SED), light curves and models. SED from radio to the very high energy gamma-rays typically shows two main bumps at low and high energies, observed in both low and high states (Figs. 5 and 6). 


\subsection{VHE emission scenarios}

Two families of scenarios have been developed to explain the VHE emission. They both require efficient mechanisms of particle acceleration at work in AGN. In leptonic scenarios, a population of relativistic electrons/positrons in a magnetized plasma produces the low-energy bump through its synchrotron emission, and the high-energy bump by Inverse-Compton effect on its own synchrotron radiation (Synchrotron-Self-Compton model, SSC) or on external radiation fields in the AGN environment (External-Compton model, EC). In hadronic scenarios, a population of very energetic hadrons interacting with matter or photons generates secondary particles, especially $\pi^{0}$ which in turn decay into VHE gamma-rays and produce the high-energy bump.

Up to now, "basic" leptonic SSC models with a single emission zone appear quite successful. They can reproduce most of the observed SED and stationary states of TeV blazars for datasets of average quality, with a number of observational constraints comparable to the number of free parameters of the models (Fig. 5). Time-dependent multi-zone SSC models can explain the HBL stationary states and even series of "flares", from the radio to the VHE range (Fig. 6). For very detailed datasets, or for radiogalaxies or non-HBL blazars (namely LBL, IBL and FSRQ), additional effects such as jet opening angle, shattered jet, jets-in-jet, or external Compton components need to be taken into account to fit the SED. Conversely, hadronic models can often reproduce the stationary states, while time-dependent hadronic models appear to be more problematic and have difficulties to explain fast variability.
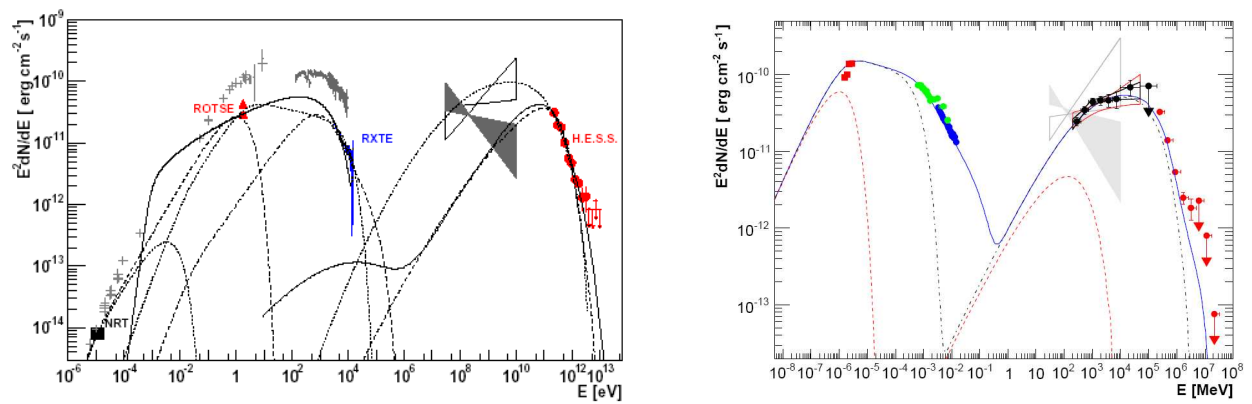

Figure 5. The quiescent state of PKS 2155-304. The SED of the source obtained at two different epochs in a stationary state can be well reproduced by basic leptonic SSC models (thin lines). Hadronic scenarios (thick line on the left panel) can also reproduce most of the TeV AGN stationary states (from [7] and [8]).
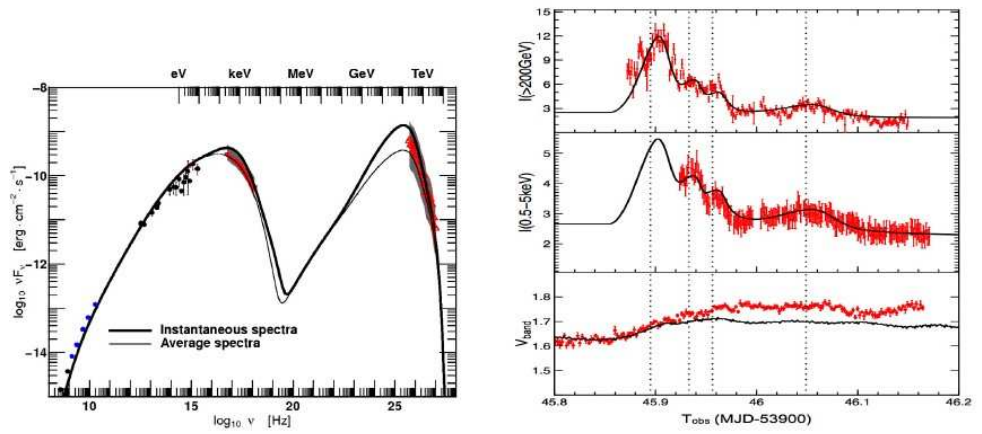

Figure 6. Time-dependent SSC model for the second flare of PKS 2155-304 in 2006 shown in Fig. 3. The model reproduces well the spectral energy distribution (left panel) and the light curves in the X-ray and gamma-ray bands (right panel) [9]. 


\subsection{Open questions}

The limits of the current emission scenarios become more apparent with the improvement of the quality of the MWL and VHE data. Available models can be seen as useful "elementary bricks" to describe the non-thermal emission, but are not yet part of a coherent global picture of the AGN population. In particular, we fail to know:

- the location, geometry and dynamics of the VHE emitting zone(s), which might be related to the black hole magnetosphere, the base of VLBI jets, the inner or outer beams and jets;

- the origin and place of the particle acceleration, namely Fermi processes in shocks and turbulence, magnetic reconnection, direct electric forces, centrifugal forces;

- the origin of high Doppler factors deduced from VHE data, despite subluminal speeds often seen by VLBI in TeV blazars (the so-called "Doppler factor crisis");

- the origin of power (accretion or rotating black hole?) and variability (accretion disk or jet physics, geometry, inhomogeneities, intermittency, instabilities?).

For all these aspects, a better understanding of the AGN non-thermal processes as a whole and of the connection between VLBI and VHE data would be mandatory.

\section{Studying the elusive radio-VHE connection in AGN: an interesting synergy between VLBI and VHE instruments}

One would expect at first glance to find correlations between the non-thermal emission and properties observed in the radio and VHE ranges since they have a common origin in the accretion-ejection process around the central engine, but amazingly it is not so. The best illustrations of such an elusive radio-VHE connection are provided by the small sample of VHE radiogalaxies discovered up to now, and by the complexity of the MWL variability of the $\mathrm{TeV}$ extragalactic sources.

\subsection{The VHE radiogalaxies}

\subsubsection{Which characteristics in radio?}

Indeed the four VHE radiogalaxies identified to date appear all quite different in the radio range. The only similarities between the two closest radiogalaxies M87, at the center of the Virgo cluster, and Cen A, a recent merger in a group of galaxies, seem to be their original classification as Wide Double Sources with a double large-scale radio structure, and the significant misalignment angle observed between their inner and extended jets. At inner scales, M87 shows a complex pattern of superluminal motion in its one-sided jet, while Cen A shows a counterjet. The two other VHE radiogalaxies are in the Perseus cluster. NGC1275, the dominant galaxy at the center of the cluster, is a core-dominated compact radiosource classified as a compact symmetric object (CSO), which is likely a young or embedded radiogalaxy. Subluminal expansion of its radio lobes has been detected with VLBI. Conversely, IC310 has been first classified in radio as a narrow angle tail or a head-tail galaxy, with a weak nuclear radio flux. Recent VLBI data re-examined that view and concluded to a blazar-like one-sided 
core-jet structure at intermediate angle to the line of sight [10]. The main VLBI characteristics of the four VHE radiogalaxies are therefore quite heterogeneous. However, it is worth noting that the detection of various possible signatures of transient BL Lac-type events has been reported in the literature for the four of them. In our current AGN unification scheme, this suggests interpreting VHE radiogalaxies as BL Lac objects with intermediate viewing angle and moderate Doppler boosting, and identifying them to the transition population between genuine beamed BL Lac objects and unbeamed FR I radiogalaxies, which could solve the long-standing question of "missing BL Lacs" in unification scenarios. High-quality VLBI and VHE datasets are urgently needed to investigate that option in depth.

\subsubsection{The MWL variability of M87}

The case of M87 is the best case investigated at the moment, with regular VLBI monitoring and several MWL campaigns from radio to VHE. However, the results have remained mostly puzzling up to now, still pending a global understanding of the compact source and parsec-scale jet. The poor astrometry of VHE data is not accurate enough to directly identify the location of the VHE emitting zone, but detection of VHE variability on daily timescales (Fig. 4) limits the possibilities to three distinct zones, namely the peculiar knot HST-1 at $\sim 65 \mathrm{pc}$ from the nucleus, the inner VLBI jet, and the central core with the black hole magnetosphere. Accordingly, three significantly different families of models have been developed corresponding to these possible zones. Then, one would expect to recognize the true VHE zone from coordinated MWL monitoring, especially thanks to the angular resolution of VLBI techniques.
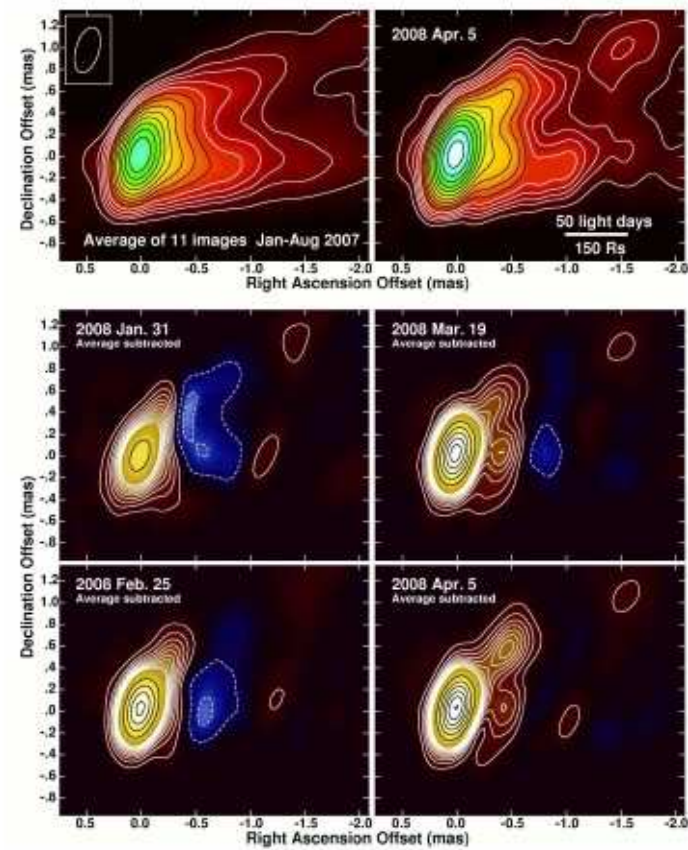

Figure 7. Monitoring of the core of M87 by the VLBA at $43 \mathrm{GHz}$. Here 0.5 mas $\sim 0.04$ pc $\sim 140 \mathrm{R}_{\mathrm{S}}$ (from [13]).

However, the complex MWL behavior of the source has prevented any firm conclusion so far. A correlation between a VHE flare and an X-ray outburst of HST-1 has been found during a 
MWL campaign in 2005. Conversely, light curves of HST-1 obtained by the Chandra satellite in 2008 do not follow the TeV ones, and it is then the radio and X-ray emission from the M87 core which is correlated to the VHE flux [11,12]. An ambitious programme of monitoring of the core of M87 by the VLBA at $43 \mathrm{GHz}$ every five days has detected a significant rise in the VLBI core at the time of VHE activity and enhanced emission along the inner jet in 2008 (Fig. 7). However, no enhancement of the radio flux in the inner region was found during significant VHE activity in 2010. On the other hand, a detailed monitoring of the kinematics of HST-1, with 26 VLBI epochs in 2006-2011 obtained with the VLBA and the EVN, concludes that VLBI structural changes of HST-1 could be related to VHE events [14]. Therefore, it has been impossible to characterize any unique common MWL and VLBI signature of VHE flares for the time being. This suggests the existence of different types of VHE events, and possibly different VHE emitting zones. However, it mostly emphasizes the present lack of a global unifying view of the various mechanisms at work.

\subsection{The case of nearby VHE blazars}

MWL monitoring of VHE blazars remains so far very difficult to interpret. Correlations appear for some events and for some spectral bands, but not for others. Even inside the same source, the MWL correlations with VHE can be different for different epochs. Moreover, the existence of orphan VHE flares seems confirmed. Here we illustrate this intricate situation with the examples of two well-known HBL sources.

\subsubsection{The northern BL Lac object Mrk 421}

Wishing to start studying the VLBI-VHE connection in the few TeV BL Lac sources known at the time, we obtained three VLBI epochs of Mrk 421 in March-April 1998 to analyze the possibility of short timescale evolution. The VLBI data showed strong evidence of complex variability in total and polarized fluxes of the radio core, at a time of increasing $\mathrm{TeV}$ activity, with one VHE flare, which suggested a correlation between TeV activity and VLBI core variability (Fig. 8). Moreover, the observed VLBI core flux appeared to be compatible with the self absorbed radio counterpart of the SSC emission at VHE [15].

These initial results motivated the launch in 2001 of a long-term radio monitoring program in Torun and Nançay, coordinated with a program at very high energies using CAT. We found a well-defined radio outburst associated with a VHE flare detected by HEGRA, with an X-ray counterpart visible in the X-ray data archive of RXTE. As shown in Fig. 9, that MWL active event was well fitted by simple SSC modeling [16] and, up to our knowledge, provided the first evidence for a firm radio-VHE connection in the light curves of a HBL object, the radio outburst being easily interpreted as the low-frequency counterpart of the SSC VHE flare.

However, more recent MWL and VLBA campaigns on Mrk 421 in 2006-2008 detected bright VHE flares, with variability detected in all other bands except in radio! No correlation between the $\mathrm{TeV}$ and radio fluxes was found. Nevertheless, small structural changes are apparent in the MOJAVE $15 \mathrm{GHz}$ VLBI images $[17,18]$. A new VLBA monitoring campaign has now started to clarify such issues and study in depth the geometry and kinematics of the jet and the VHE zone, the Doppler factor, the variability and the non-thermal emission processes (Fig.10) [19]. 

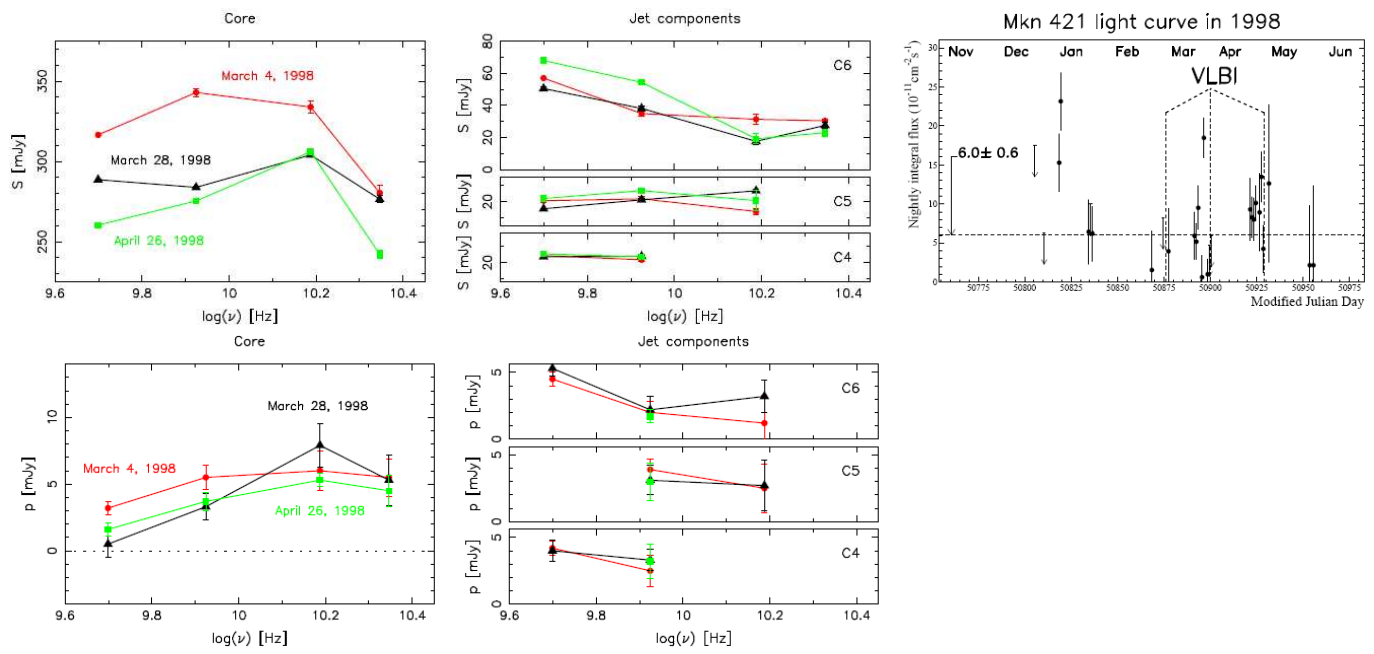

Figure 8. Complex evolution of total and polarized spectra of the VLBI core and jet components in Mrk 421, studied at three epochs in 1998 (left panel), together with a monitoring by CAT at VHE (right panel). See [15] for further comments.

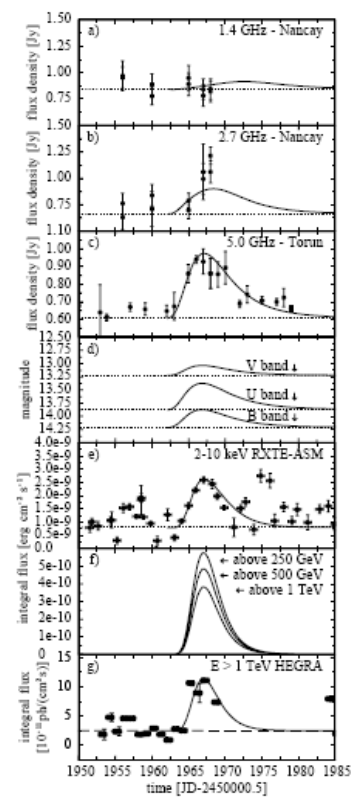

Figure 9. Light curves in various spectral ranges showing a flare of Mrk 421 observed in 2001 in radio, X-rays and VHE by Torun and Nançay, RXTE, and HEGRA. This MWL active event is well reproduced by a simple SSC scenario (full lines) [16].

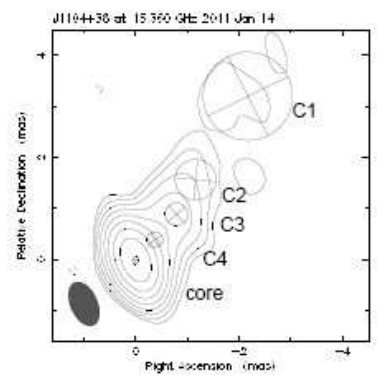

Figure 10. Recent VLBI monitoring of Mrk 421 provides a detailed description of the apparent subluminal motion of components $\mathrm{C} 4$ to $\mathrm{C} 2$, and an apparent motion of $(1.3 \pm 0.5)$ c for component $\mathrm{C} 1$ [19]. 


\subsubsection{The southern BL Lac object PKS 2155-304}

As shown in Fig.11, the source PKS 2155-304 offers a striking example of a long term increase of its radio flux over months, starting at the time of a spectacular activity in the VHE range, with two intense and highly variable flares in 2006 (Fig. 3).

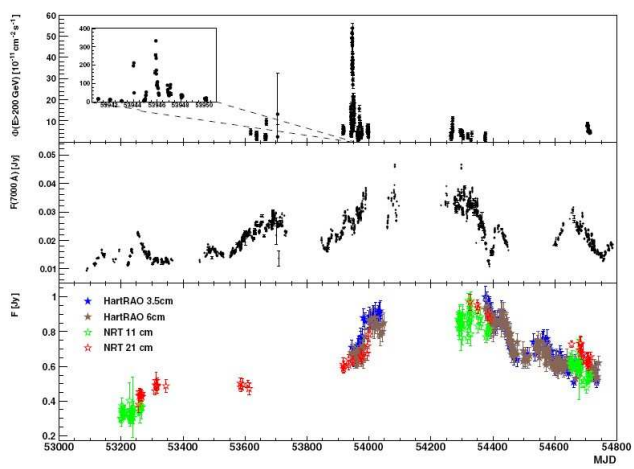

Figure 11. Long-term light curves of PKS 2155-304 in the VHE, optical and radio ranges. Radio data were gathered from Nançay, HartRAO and ATCA. An extremely active state is seen at VHE just at the beginning of a long-term increase of the radio fluxes, bearing some clues for a radio-VHE connection [9].

Although the flares observed in X-rays and gamma-rays are well reproduced by SSC models as illustrated in Fig. 6, the outburst observed in the radio range is too long and too high to be simply interpreted as the radio counterpart of the high energy flares. Only the development of successful global scenarios locating the VLBI core, the jet components and the VHE zone respectively one to each other, will provide convincing explanations for such MWL behavior.

\section{Next generation of ground-based astronomy: the Cherenkov Telescope Array (CTA) project}

Exploring the non-thermal universe will benefit from a significant synergy between present and future radio and VHE instruments, at the two extreme parts of the electromagnetic spectrum, with the large scale infrastructures of astrophysics and astroparticle physics as LOFAR and SKA and its precursors on one side, and CTA, HAWC and LHAASO on the other side. The elusive connection between the radio and VHE properties of $\mathrm{TeV}$ active galactic nuclei clearly exists but is not easy to catch and interpret. It will require gathering VHE data in coordination with radio data on all time scales, in total and polarized fluxes, and with VLBI angular resolution. This might require long-term monitoring over a few years and raise the question how to optimize the observational strategy and limit the observing time.

The CTA project aims to improve by a factor 10 the sensitivity at $\mathrm{TeV}$ energies, while also significantly improving the effective energy coverage, the field of view and the angular resolution. Full sky coverage is foreseen with two arrays in the southern and northern hemispheres. One possible configuration for the future southern array consists in a low-energy section with $4 \times 23 \mathrm{~m}$ parabolic telescopes (LST) above a few $10 \mathrm{GeV}$, a core-energy array with $23 \times 12 \mathrm{~m}$ Davies-Cotton telescopes (MST) in the range $100 \mathrm{GeV}-10 \mathrm{TeV}$, and a high-energy section with $32 \times 5-6 \mathrm{~m}$ Davies-Cotton or Schwarzschild-Couder telescopes at multi-TeV energies (Fig. 12). Various geometries are currently studied in order to optimize the array performances (Figs. 12 and 13). 

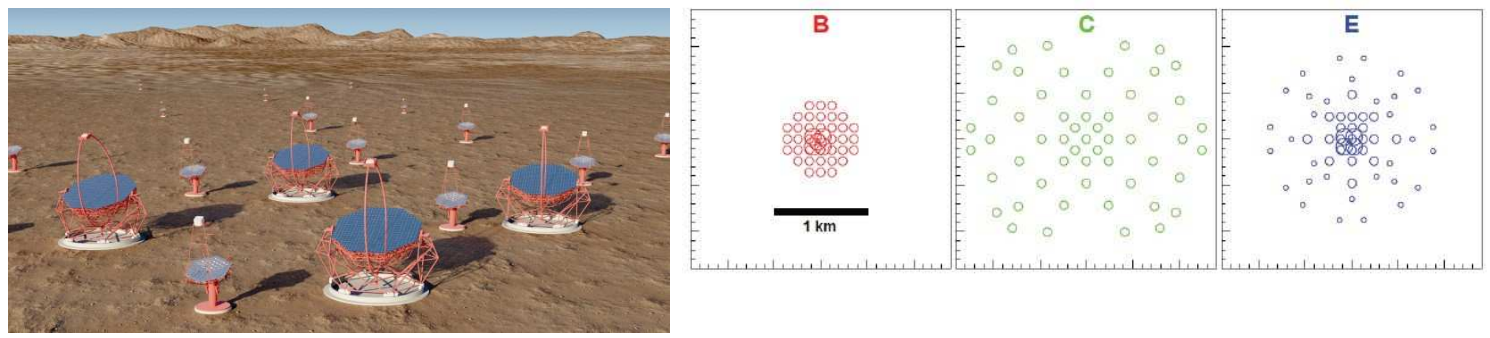

Figure 12. The CTA project. Left: CTA will consist in several tens of telescopes of various types, with large (LST), medium (MST) and small (SST) size telescopes. Right: Various options are under studies for the array, a densely packed array of LST and MST focused on low energies (left panel), a wide spread array of MST focused on mid and high energies (middle panel), and a mixed array of SST, MST and LST providing a more balanced sensitivity over a large energy range (right panel) [20].

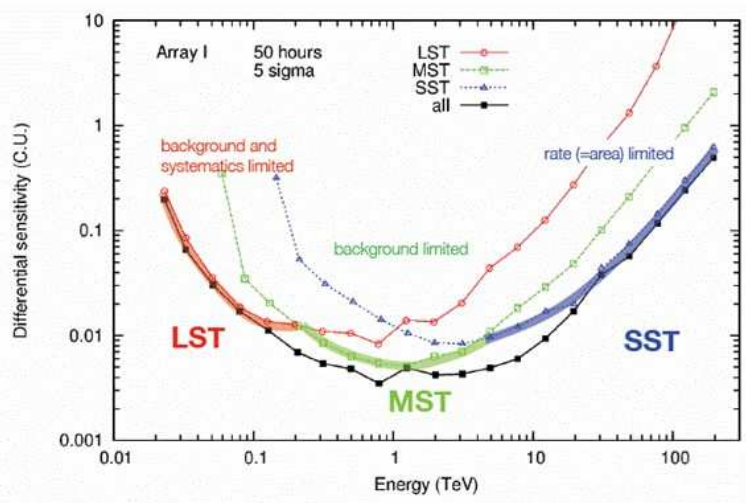

Figure 13. Differential sensitivity of a typical mixed array for CTA from a few $10 \mathrm{GeV}$ to more than $100 \mathrm{TeV}$, for an observing time of 50 hours and detection limit at $5 \sigma$. Sensitivity curves are also shown for the sub-arrays of LST, MST and SST (from [20]).

The performances expected from CTA should allow the instrument reaching several hundreds of $\mathrm{TeV}$ active galactic nuclei of various types, and monitoring AGN samples on short and long time scales. It should be also possible to detect the quiescent VHE states in several sources. VHE intermittent flares could be triggered at various places, from black hole magnetospheres to relativistic beams and jets, as long as they benefit from Doppler boosting, which could explain the variety of time scales, sizes and correlations observed. VLBI data will examine how important the relativistic beaming in VHE AGN is and whether it is decisive or not for successful VHE detection. Regular VLBI mapping coordinated with CTA campaigns should clarify the nature of the correlation between the two spectral ranges and significantly constrain the global picture of the AGN central core at the sub-parsec scale, linking the mechanism of jet formation, the ejection of VLBI knots, the growth of shocks and turbulence, together with the localization, geometry, dynamics and evolution of the VHE emitting zones.

\section{Conclusion}

VHE observations allow exploring a new specific facet of the AGNs, related to extreme processes and high variability, not yet self-consistently understood in its global environment. Coordinated VLBI data could be decisive especially to fix the location, geometry and dynamics of the regions emitting the VHE quiescent emission and flares, and together with 
MWL monitoring, to cover the missing link between accretion, black hole magnetosphere and ejection, possibly detecting emission from outflows from the disk and magnetosphere and from relativistic beams streaming along inner jets.

\section{References}

[1] Sol, H., Zech, A., Boisson, C., et al., Active Galactic Nuclei under the scrutiny of CTA, Astropart. Phys., http://dx.doi.org/10.1016/j.astropartphys.2012.12.005 (2013)

[2] Becherini, Y., Boisson, C., Cerruti, M., and the HESS collaboration, AIPC, 1505, 490 (2012)

[3] Pita, S., Goldoni, P., Boisson, C., et al., AIPC, 1505, 566 (2012)

[4] Abramowski, A., Acero, F., Aharonian, F., et al., A\&A, 520, A83 (2010)

[5] Albert, J., Aliu, E., Anderhub, H., et al., ApJ, 669, 862 (2007)

[6] Aharonian, F., Akhperjanian, A. G., Bazer-Bachi, A. R., et al., Science, 314, 1424 (2006)

[7] Aharonian, F., Akhperjanian, A. G., Anton, G., et al., ApJL, 696, L150 (2009)

[8] Aharonian, F., Akhperjanian, A. G., Bazer-Bachi, A. R., et al., A\&A, 442, 895 (2005)

[9] Abramowski, A., Acero, F., Aharonian, F., et al., A\&A, 539, A149 (2012)

[10] Kadler, M., Eisenacher, D., Ros, E., et al., A\&A, 538, L1 (2012)

[11] Acciari, V.A., Aliu, E., Arlen, T., et al., Science, 325, 444 (2009)

[12] Harris, D. E., Cheung, C. C., Stawarz, Lukasz, Biretta, J. A., Perlman, E. S., ApJ, 699, 305 (2009)

[13] Abramowski, A., Acero, F., Aharonian, F., et al., ApJ, 746, 151 (2012)

[14] Giroletti, M., Hada, K., Giovannini, G., et al., A\&A, 538, 10 (2012)

[15] Charlot, P., Gabuzda, D., Sol, H., Degrange, B., Piron, F., A\&A, 457, 455 (2006)

[16] Katarzynski, K., Sol, H., Kus, A., A\&A, 410, 101 (2003)

[17] Acciari, V.A., Aliu, E., Arlen, T., et al., ApJ, 738, 25 (2011)

[18] Aleksic, J., Alvares, E. A., Antonelli, L. A., et al., A\&A, 542, A100 (2012)

[19] Lico, R., Giroletti, M., Orienti, M., et al., A\&A, 545, 117 (2012)

[20] Bernlohr, K., Barnacka, A., Becherini, Y., et al., Astropart. Phys., 43, 171 (2013) 